\title{
Differing Serum Cea in Primary and Recurrent Rectal Cancer - A Reflection of Histology?
}

\author{
Angela C Chang ${ }^{\mathrm{a}, \mathrm{b}}$, Leigh R Warren ${ }^{\mathrm{a}, \mathrm{b}}$, Savio G Barreto ${ }^{\mathrm{a}, \mathrm{c}}$, Randolph Williams ${ }^{\mathrm{a}}$
}

\begin{abstract}
Background: Serum carcinoembryonic antigen (CEA) levels are not universally measured in colorectal cancers. CEA levels have been reported to be usually normal at time of primary rectal cancer diagnosis but elevated in recurrent disease. The aims of the study were to (1) compare serum CEA levels performed at time of primary and recurrent colorectal tumour diagnosis; and (2) to determine serum CEA levels in rectal cancers at primary diagnosis to analyse potential factors influencing differing CEA levels.
\end{abstract}

Methods: A retrospective analysis of patients treated for colorectal cancers at Modbury Hospital, South Australia was performed. Each admission was reviewed within the electronic database. Serum CEA levels and tumour-related factors were determined in patients who underwent curative surgery for their primary tumour and developed tumour recurrence/metastases within the study period.

Results: 438 patients were treated for colorectal cancer in the study period. In patients who underwent curative surgery and developed a recurrence, serum CEA was elevated in $20 \%$ patients at primary diagnosis and in $46.6 \%$ patients at recurrence. Only 1 of 30 patients with rectal cancer had an elevated CEA at diagnosis of primary tumour. Tumour relationship to the peritoneal reflection did not appear to play a role.

Conclusions: In rectal cancers, serum CEA levels are often normal

Manuscript accepted for publication March 23, 2012

${ }^{a}$ Department of Surgery, Modbury Hospital, South Australia, Australia

${ }^{\mathrm{b}}$ These authors contributed equally to this manuscript

${ }^{\mathrm{c}}$ Corresponding author: Savio G Barreto, Department of Surgery,

Modbury Hospital, Smart Road, Modbury, Australia.

Email: georgebarreto@yahoo.com

doi:10.4021/wjon479w at the time of initial diagnosis. However, this should not preclude its use in post-operative surveillance. Serum CEA levels noted in primary rectal cancer appear unrelated to the relationship of the tumour to the peritoneal reflection. Stroma-related factors could possibly be involved and merit further investigation.

Keywords: Cancer; CEA; Colorectal; Rectal

\section{Introduction}

Carcinoembryonic antigen (CEA) is a tumour marker widely measured in colorectal cancer (CRC) [1] at time of primary cancer diagnosis and as part of the intensive surveillance [24]. This has led to improved outcomes [2, 3, 5]. Although recommended in many countries $[1,6]$, Australia's previous National Health and Medical Research Council (NHMRC) guidelines [7] did not favour routine measurement of CEA for all CRC patients. The current guidelines do recommend its performance [8]. The presence of a normal preoperative serum CEA has confounded the use of CEA in follow-up in other countries [9].

Patients with rectal tumours are less likely to present with a raised serum CEA level. Grossmann et al [10] observed that in patients with primary rectal cancer and a normal CEA, CEA became elevated in up to $50 \%$ on follow-up in the presence of recurrent disease. The authors did not provide a clear hypothesis for this observation.

The aims of the current study were to 1) compare the serum CEA levels at time of primary and recurrent CRC diagnosis and 2) determine serum CEA levels in rectal cancers at primary diagnosis, as per Grossmann et al [10], and to identify potential factors influencing differing CEA levels.

\section{Material and Methods}

A retrospective search of a prospectively maintained electronic database of a public teaching hospital was undertaken. International Classification of Diseases (ICD) codes for colon and rectum cancers for a 58-month period, from January 
Table 1. Data of the 15 Patients With CEA Levels Available at the Time of Primary Tumour and Recurrence

\begin{tabular}{|c|c|c|c|c|c|c|}
\hline \multicolumn{2}{|c|}{ Patient Demographics } & \multicolumn{3}{|c|}{ Primary Cre } & \multicolumn{2}{|c|}{ Recurrent Crc } \\
\hline Gender & Age & CEA & Location & Stage & CEA & Location \\
\hline \multicolumn{7}{|c|}{ CEA normal primary $\mathrm{CRC} \rightarrow \mathrm{CEA}$ normal recurrent colorectal carcinoma } \\
\hline Male & 64 & 2.3 & High rectum & $3 b$ & 7 & Liver \\
\hline Male & 72 & 1 & Sigmoid & $2 \mathrm{a}$ & 1 & Liver \\
\hline Female & 56 & 8 & High rectum & $2 b$ & 2 & Pelvic wall \\
\hline Male & 84 & 2 & Transverse colon & $3 b$ & 4 & Brain, lungs \\
\hline Male & 78 & 2 & Descending colon & $3 \mathrm{c}$ & 1 & Liver, spleen \\
\hline Male & 70 & 3.4 & Caecum & $3 b$ & 1 & Brain \\
\hline Female & 77 & 3 & Sigmoid & $3 c$ & 2 & Liver \\
\hline
\end{tabular}

CEA normal primary $\mathrm{CRC} \rightarrow \mathrm{CEA}$ elevated recurrent colorectal carcinoma

\begin{tabular}{ccccccc}
\hline Male & 64 & 2.3 & Low rectum & $3 \mathrm{~b}$ & 98 & Lung \\
Female & 80 & 1.6 & Caecum & $2 \mathrm{~b}$ & $6.3 *$ & Liver \\
Male & 69 & 1 & Low rectum & $2 \mathrm{a}$ & 52 & Local \\
Male & 80 & 1 & Low rectum & $2 \mathrm{a}$ & 17 & Liver \\
\hline
\end{tabular}

CEA elevated primary $\mathrm{CRC} \rightarrow \mathrm{CEA}$ elevated recurrent colorectal carcinoma

\begin{tabular}{ccccccc}
\hline Female & 72 & 39.3 & Rectosigmoid & $3 b$ & 180.9 & Liver \\
Male & 73 & 15 & Rectosigmoid & $3 b$ & 11 & Liver, lung \\
Male & 73 & 211.8 & Transverse colon & $3 \mathrm{c}$ & 6975 & Lung \\
\hline
\end{tabular}

CEA elevated primary $\mathrm{CRC} \rightarrow \mathrm{CEA}$ normal recurrent colorectal carcinoma

Female $60 \quad 14.4 \quad$ Caecum
$\begin{aligned} & \text { * The normal range for CEA was defined as }<5 \mu \mathrm{g} / \mathrm{L} \text { by the laboratory in which this patient underwent her test as opposed } \\ & \text { to }<10 \mu \mathrm{g} / \mathrm{L} \text { in the other patients. }\end{aligned}$

2007 to October 2011 were analysed with the aim of identifying all patients treated for colorectal cancer at the hospital. The overall cohort included patients who: a) Underwent surgery - for primary tumour or recurrence; b) Underwent chemotherapy - adjuvant or palliative, or; c) Received palliative care - for metastatic disease or local recurrence, at the hospital in the study period.

Each admission was reviewed within the electronic database. The case records of patients identified to have a primary tumour and a tumour recurrence within the stated study period were manually reviewed.

\section{Variables collected for each patient}

Age, sex, serum CEA level $(\mu \mathrm{g} / \mathrm{L})$ at diagnosis of primary tumour and/or at time of recurrence, surgery, tumour histology, and primary and metastatic tumour location. Each tumour was additionally restaged based on the revised American Joint Committee on Cancer (AJCC) staging and the International Union against Cancer (UICC) TNM staging [11].

Inclusion criteria for evaluation of CEA in recurrent disease 
Table 2. Data of the 30 Patients With CEA Levels Available at the Time of Primary, Non Metastatic Rectal Cancer Based on Tumour Relation to the Reflection of the Peritoneal Fold

\begin{tabular}{llll}
\hline Tumour location & CEA normal & CEA elevated & Total patients \\
\hline High & 12 & 1 & 13 \\
Low & 17 & 0 & 17 \\
\hline
\end{tabular}

CEA: Carcinoembryonic antigen.

Patients who underwent curative surgery for their primary tumour and developed tumour recurrence/metastases within the study period.

\section{Inclusion criteria for evaluation of CEA in primary rec- tal cancer and definitions}

Patients with primary, non-metastatic (distant) rectal cancer within the study period. Lymph node involvement on histology was not considered metastatic.

For the analysis of CEA levels in primary rectal cancer, the peritoneal reflection [12] was regarded as the landmark dividing the high and the low rectum based on the biologic barrier between lymphatic pathways. Rectal adenocarcinomas above the reflection were classified as 'high' while rectal and anal canal adenocarcinomas below the reflection were classified as 'low'. Anal canal adenocarcinomas were grouped with rectal cancers owing to a previous study indicating a similar CEA expression profile between these two sites [13]. For the purpose of this study, 'recurrent' cancer refers to local recurrence and distal metastases.

This was a retrospective study. No experiments we conducted on human or animal subjects. Identifying patient details were available to the study investigators only.

\section{Statistical analysis}

The data was compiled and analysed using Microsoft Excel 2007 (Microsoft, Redmond, California). Where applicable, data has been provided as median (range).

\section{Results}

A total of 438 patients were treated for different stages of colorectal cancers at the hospital during the study period. These included 233 male patients $(53.2 \%)$ and 205 female patients $(46.8 \%)$. The median age was 72 years (range: 21 - 102).

\section{CEA in Recurrent disease}

Of the 438 patients, 46 patients $(10.5 \%)$ underwent curative surgery for a primary tumour and developed tumour recurrence/metastases within the study period thereby fulfilling the criteria for inclusion. Of these, only 15 patients had CEA levels measured at the time of primary and recurrent cancer diagnosis. The data of these patients has been summarised in Table 1 . CEA was elevated in only 3 patients $(20 \%)$ with primary colorectal cancers and in 7 patients $(46.6 \%)$ with recurrent or metastatic cancers.

\section{CEA in rectal cancer}

Of the overall colorectal cancer cohort of 438 patients, 79 patients were treated for rectal cancers. This included 50 male patients $(63 \%)$ and 29 female patients $(36.7 \%)$ with a median age of 67 years (range: 39 - 102).

Of the 79 patients, relevant data on the CEA levels was available in 39 patients $(49.3 \%)$. Of the 39 patients, 30 patients had primary, non-metastatic (distant) rectal cancer and were included for further analysis.

Table 2 shows the rectal tumour location and CEA level of these patients.

\section{Discussion}

The data from our study indicates that CEA is of more value in detecting recurrent rather than primary colorectal cancers amongst the cohort of patients who underwent primary curative surgery and developed a recurrence, and in whom CEA levels were available for comparison at the two time points within the study period. There was no obvious correlation between the stage of primary disease and serum CEA levels in this data. The findings from this study were consistent with those of Grossmann et al [10] that while primary rectal cancers, unlike colon cancers, may not be accompanied by an elevated serum CEA level, CEA levels may be elevated in recurrent disease. This supports the routine use of serum CEA in follow-up protocols of patients with rectal cancers. Our data did not indicate a correlation between the anatomical relationship of the tumour to the peritoneal reflection. 
The latter inference indicates that there must be other reasons for the differential expression of serum CEA in primary and recurrent rectal cancers. In 1993, $\mathrm{Ng}$ et al [14] noted a significant positive correlation between CEA imunoperoxidase staining in the basolateral regions of cells and stroma of colorectal cancers and elevated plasma CEA levels. We hypothesized that the difference in serum CEA levels between colon and rectal cancer may be related to differing factors in their stroma.

In order to explain the lack of elevation in serum CEA in primary rectal cancers, we thus sought to determine if there existed any such differences between the colonic and rectal tissue.

Multinucleated stromal giant cells are relatively more abundant in the colonic stroma [15] as opposed to the rectum. When involved in carcinomas of the breast, these cells have been shown to stain positively for CEA [16]. Possibly these cells are involved in the expression of serum CEA at other sites. These multinucleate giant cells express matrix metalloproteinase-9 (MMP-9) which is responsible for the accelerated breakdown of the extracellular matrix associated with tumour invasion and metastases [17].

Another potentially relevant difference in stroma between the colon and rectum lies in the distribution of myofibroblasts. Myofibroblasts have been shown to regulate a number of tumour-promoting functions, including angiogenesis, invasion and metastasis and are generally located at the invasive front of tumours [18]. More than $80 \%$ of the fibroblast-like cells located at the leading edge of colon cancer were found to be myofibroblasts [19]. This is in stark contrast to the rectal cancer where myofibroblasts are less extensively distributed in the more prevalent intermediate and mature fibrotic stroma.

CEA is an E- and L-selectin ligand and hence may play a role in metastasis [20]. It is possible that the differing stromal factors between colon and rectal cancers may account for some of the differences in serum CEA levels between cancers located in the two sections of the large bowel.

An elevation in CEA accompanying the recurrence or metastasis of rectal cancer in cases where the CEA was normal in the primary tumour can also be explained, at least in part, by the role of myofibroblasts. Recent data has provided evidence that in the case of metastases, for instance in the liver, myofibroblasts develop from hepatic stellate cells native to the metastatic site [21] and not from the primary tumour.

One of the limitations of our study is the small number of patients in the study cohort despite a large denominator in terms of the overall cohort. This suggests that serum CEA was not performed regularly for many of the patients in the initial cohort. While this could potentially reflect the older recommendations from the NHMRC in which performance of CEA was not considered mandatory, it could also simply indicate the resultant individual clinicians practice protocols.

\section{Conclusions}

Measuring serum CEA is more useful in post-operative surveillance of colorectal cancer compared to performance at the time of primary tumour diagnosis. In rectal cancers, normal serum CEA levels are commonly encountered at the time of diagnosis of the primary tumour. However, this should not preclude its use in the post-operative surveillance. Serum CEA levels noted in primary rectal cancer appear unrelated to the relationship of the tumour to the peritoneal reflection, although, stroma-related factors could possibly be involved and merit further investigation.

\section{Acknowledgement}

Thank you to Martin Moyse for his assistance with data collection.

\section{Conflicts of Interest}

None declared (all authors).

\section{Author Contributions}

Angela Chang: Design, definition of intellectual content, literature search, data acquisition, data analysis, statistical analysis, manuscript editing and manuscript review. Leigh Warren: Design, definition of intellectual content, literature search, data acquisition, data analysis, manuscript editing and manuscript review. Savio Barreto: Guarantor. Concept, design, definition of intellectual content, literature search, data acquisition, data analysis, statistical analysis, statistical analysis, manuscript preparation, manuscript editing and manuscript review. Randolph Williams: Design, interpretation of data, manuscript editing and manuscript review.

\section{References}

1. Duffy MJ, van Dalen A, Haglund C, Hansson L, Holinski-Feder E, Klapdor R, Lamerz R, et al. Tumour markers in colorectal cancer: European Group on Tumour Markers (EGTM) guidelines for clinical use. Eur J Cancer. 2007;43(9):1348-1360.

2. Bruinvels DJ, Stiggelbout AM, Kievit J, van Houwelingen HC, Habbema JD, van de Velde CJ. Follow-up of patients with colorectal cancer. A meta-analysis. Ann Surg. 1994;219(2):174-182.

3. Figueredo A, Rumble RB, Maroun J, Earle CC, Cummings B, McLeod R, Zuraw L, et al. Follow-up of pa- 
tients with curatively resected colorectal cancer: a practice guideline. BMC Cancer. 2003;3:26.

4. Jeffery M, Hickey BE, Hider PN. Follow-up strategies for patients treated for non-metastatic colorectal cancer. Cochrane Database Syst Rev. 2007(1):CD002200.

5. Renehan AG, Egger M, Saunders MP, O'Dwyer ST. Impact on survival of intensive follow up after curative resection for colorectal cancer: systematic review and meta-analysis of randomised trials. BMJ. 2002;324(7341):813.

6. Locker GY, Hamilton S, Harris J, Jessup JM, Kemeny N, Macdonald JS, Somerfield MR, et al. ASCO 2006 update of recommendations for the use of tumor markers in gastrointestinal cancer. J Clin Oncol. 2006;24(33):53135327.

7. National Health and Medical Research Council [http:// www.nhmrc.gov.au]. Canberra; c1999-2005 [updated August 5th 2011, cited December 22 2011]. Australian Government; [PDF available for download]. Available at http://www.nhmrc.gov.au/_files_nhmrc/publications/ attachments/cp62.pdf.

8. Australian Cancer Network Colorectal Cancer Guidelines Revision Committee. Guidelines for the Prevention, Early Detection and Management of Colorectal Cancer. The Cancer Council Australia and Australian Cancer Network, Sydney 2005.

9. Grossmann I, de Bock GH, van de Velde CJ, Kievit J, Wiggers T. Results of a national survey among Dutch surgeons treating patients with colorectal carcinoma. Current opinion about follow-up, treatment of metastasis, and reasons to revise follow-up practice. Colorectal Dis. 2007;9(9):787-792.

10. Grossmann I, de Bock GH, Meershoek-Klein Kranenbarg WM, van de Velde CJ, Wiggers T. Carcinoembryonic antigen (CEA) measurement during follow-up for rectal carcinoma is useful even if normal levels exist before surgery. A retrospective study of CEA values in the TME trial. Eur J Surg Oncol. 2007;33(2):183-187.

11. Sobin L, Gospodarowicz M, Wittekind C. UICC: TNM classification of malignant tumours. 7th ed. Oxford:
Wiley-Blackwell, 2009.

12. Roels S, Duthoy W, Haustermans K, Penninckx F, Vandecaveye V, Boterberg T, De Neve W. Definition and delineation of the clinical target volume for rectal cancer. Int J Radiat Oncol Biol Phys. 2006;65(4):1129-1142.

13. Tanum G, Stenwig AE, Bormer OP, Tveit KM. Carcinoembryonic antigen in anal carcinoma. Acta Oncol. 1992;31(3):333-335.

14. Ng IO, Ho J, Pritchett CJ, Chan EY, Ho FC. CEA tissue staining in colorectal cancer patients--correlation with plasma CEA, histology and staging. Pathology. 1993;25(3):219-222.

15. Wu ML, Zhao X. Multinucleated stromal giant cells in colonic lamina propria. Histopathology. 2007;50(5):584590.

16. Nielsen BB, Kiaer HW. Carcinoma of the breast with stromal multinucleated giant cells. Histopathology. 1985;9(2):183-193.

17. Ueda Y, Imai K, Tsuchiya H, Fujimoto N, Nakanishi I, Katsuda S, Seiki M, et al. Matrix metalloproteinase 9 (gelatinase B) is expressed in multinucleated giant cells of human giant cell tumor of bone and is associated with vascular invasion. Am J Pathol. 1996;148(2):611-622.

18. Conti J, Thomas G. The Role of Tumour Stroma in Colorectal Cancer Invasion and Metastasis. Cancers 2011;3:2160-68.

19. Illemann M, Hansen U, Nielsen HJ, Andreasen PA, Hoyer-Hansen G, Lund LR, Dano K, et al. Leadingedge myofibroblasts in human colon cancer express plasminogen activator inhibitor-1. Am J Clin Pathol. 2004;122(2):256-265.

20. Thomas SN, Zhu F, Schnaar RL, Alves CS, Konstantopoulos K. Carcinoembryonic antigen and CD44 variant isoforms cooperate to mediate colon carcinoma cell adhesion to E- and L-selectin in shear flow. J Biol Chem. 2008;283(23):15647-15655.

21. Badiola I, Olaso E, Crende O, Friedman SL, VidalVanaclocha F. Discoidin domain receptor 2 deficiency predisposes hepatic tissue to colon carcinoma metastasis. Gut. 2011. 